\title{
Bright and dark breathers in Fermi-Pasta-Ulam lattices
}

\author{
B. Sánchez-Rey, ${ }^{1}$ G. James, ${ }^{2}$ J. Cuevas, ${ }^{1}$ and J. F. R. Archilla ${ }^{1}$ \\ ${ }^{1}$ Nonlinear Physics Group, University of Seville, 41001 Seville, Spain \\ ${ }^{2}$ Laboratoire Mathématiques pour l'Industrie et la Physique (UMR 5640), INSA de Toulouse, 31077 Toulouse, Cedex 4, France
}

(Received 6 June 2003; revised manuscript received 5 February 2004; published 9 July 2004)

\begin{abstract}
In this paper we study the existence and linear stability of bright and dark breathers in one-dimensional FPU lattices. On the one hand, we test the range of validity of a recent breathers existence proof [G. James, C. R. Acad. Sci., Ser. I: Math, 332, 581 (2001)] using numerical computations. Approximate analytical expressions for small amplitude bright and dark breathers are found to fit very well exact numerical solutions even far from the top of the phonon band. On the other hand, we study numerically large amplitude breathers nonpredicted in the above cited reference. In particular, for a class of asymmetric FPU potentials we find an energy threshold for the existence of exact discrete breathers, which is a relatively unexplored phenomenon in one-dimensional lattices. Bright and dark breathers superposed on a uniformly stressed static configuration are also investigated.
\end{abstract}

DOI: 10.1103/PhysRevB.70.014301

PACS number(s): 63.20.Pw, 63.20.Ry

\section{INTRODUCTION AND MODEL}

Discrete breathers, also called intrinsic localized modes, are classical exact spatially localized time-periodic solutions which can be sustained by many nonlinear lattices (see Refs. 1 and 2 for a review). In 1994 MacKay and Aubry ${ }^{3}$ rigorously proved their existence in Hamiltonian lattices with anharmonic on-site potentials and weak coupling. Breathers are obtained by continuation from the uncoupled case in which trivial breathers exist (e.g., breathers with only one oscillator excited, the others being at rest). With the same technique, the existence of breathers was also proved for diatomic Fermi-Pasta-Ulam (FPU) chains. ${ }^{4}$ In this model, two different masses alternate on the chain and are nonlinearly coupled to their nearest neighbors via an interaction potential $V$. This result is valid for a large mass ratio, since light masses (nextnearest neighbors) are weakly coupled due to the presence of a heavy mass in between.

Unfortunately this method is not applicable to homogeneous FPU lattices, which do not possess an uncoupled limit in which trivial breathers exist. For some years the only existence result concerned the particular potentials $V(x)=x^{2 m}$ with $m \geqslant 2 .{ }^{5}$ FPU lattices seemed elusive to a rigorous mathematical treatment in spite of numerous approximate ${ }^{6-8}$ and numerical ${ }^{9,10}$ studies, which have indicated the existence of discrete breathers in these systems.

Nevertheless, recent papers have presented rigorous existence proofs for discrete breathers in infinite FPU lattices. On the one hand, Aubry et al. ${ }^{11}$ have proved the existence of breathers with frequencies above the phonon spectrum, when $V$ is a strictly convex polynomial of degree 4 . These results are obtained via a variational method and apply in fact to higher dimensional generalizations of FPU lattices. Without additional assumptions on $V$, these results give only a partial information on breathers amplitudes. Under the additional condition that $V$ is even, Aubry et al. prove the existence of breathers of arbitrarily small amplitudes in one-dimensional FPU lattices.

On the other hand, the existence (respectively, nonexistence) of small amplitude breathers with frequencies slightly above the phonon band has been established when $V$ satisfies (respectively, violates) a local hardening condition. ${ }^{12}$ The proof is based on a center manifold technique, which shows more generally that all small amplitude time-periodic solutions of the FPU system (including breathers) are determined by a two-dimensional map, provided their frequency lies near the phonon band edge. The discrete center manifold method has been put in a general framework ${ }^{13}$ and applied to other systems, namely diatomic FPU chains ${ }^{14}$ (far from the uncoupled regime) and spin lattices. ${ }^{15}$

The aim of this work is to test numerically the range of validity of the center manifold method ${ }^{12}$ and to explore new phenomena, far from the small amplitude regime.

The one-dimensional FPU system is given by the following equations:

$$
\ddot{x}_{n}=V^{\prime}\left(x_{n+1}-x_{n}\right)-V^{\prime}\left(x_{n}-x_{n-1}\right), \quad n \in \mathbb{Z},
$$

where $x_{n}$ represents mass displacements from their equilibrium positions and $V$ is a smooth interaction potential satisfying $V^{\prime}(0)=0, V^{\prime \prime}(0)=1$. In Ref. 12, the existence of small amplitude breathers (SAB) with frequencies $\omega_{b}$ slightly above the phonon band $\left(\omega_{b}>2\right)$ is obtained for $B>0$, where

$$
B=\frac{1}{2} V^{(4)}(0)-\left(V^{(3)}(0)\right)^{2},
$$

and their nonexistence is proved for $B<0$ (see Refs. 16 and 17 for related results on the modulational instability of nonlinear normal modes and the tangent bifurcation of standing waves, respectively).

The parameter $B$ can be interpreted as a hardening coefficient, since breathers with amplitude $A \approx 0$ have frequency $\omega_{b} \approx 2+(B / 8) A^{2}$. Note that $B$ is slightly different from the classical hardening coefficient of an anharmonic potential $[V$ is hard if $\frac{3}{5} V^{(4)}(0)-\left(V^{(3)}(0)\right)^{2}>0$ and soft for $\frac{3}{5} V^{(4)}(0)$ $\left.-\left(V^{(3)}(0)\right)^{2}<0\right]$.

In this paper we consider anharmonic potentials 


$$
V(u)=\frac{u^{2}}{2}+\frac{K_{3}}{3} u^{3}+\frac{K_{4}}{4} u^{4}
$$

(at least one of the coefficients $K_{3}, K_{4}$ is nonzero). One has $B=3 K_{4}-4 K_{3}^{2}$ and thus the parameter $B$ is positive if $\left|K_{3}\right|<\sqrt{3 K_{4}} / 2$. In the case $K_{3}=0$ we have an even potential and the sign of $B$ coincides with the sign of the quartic coefficient $K_{4}$.

In Section II we compute numerically $\mathrm{SAB}$ and find that they are very well fitted by leading order analytical expressions deduced from Ref. 12. In Section III we consider a class of potentials for which numerical computations yield large amplitude breathers (LAB) with frequencies near the top of the phonon band. In this case there is an energy threshold for the existence of exact discrete breathers. The existence of an energy threshold for breather creation in higher-dimensional lattices is well known, ${ }^{18,19}$ but only a few one-dimensional examples have been given where such a threshold exists. ${ }^{11,20,21}$ Section III explores in more details the example of Ref. 21. Section IV deals with dark breathers, i.e., spatially modulated standing waves whose amplitude is constant at infinity and vanishes at the center of the chain. Leading order analytical expressions for small amplitude dark breathers (with frequencies inside the phonon band) fit the numerically computed dark breathers very well in the case of even potentials. In Sec. V we analyze bright and dark breathers superposed on uniformly stretched or compressed static states. In each section the linear stability of the abovementioned solutions is numerically investigated.

\section{SMALL AMPLITUDE BREATHERS}

\section{A. Summary of the theory}

We consider time-periodic solutions of (1) having a given frequency $\omega_{b}$. We introduce the interaction force

$$
y_{n}=V^{\prime}\left(u_{n}\right)=u_{n}+K_{3} u_{n}^{2}+K_{4} u_{n}^{3},
$$

where

$$
u_{n}=x_{n}-x_{n-1} .
$$

The time-average of $y_{n}$ is independent of $n$ [integrate (1)], and is fixed to 0 in Ref. 12, as it is the case for all spatially localized solutions. Then, problem (1) leads to

$$
\frac{d^{2}}{d t^{2}}\left(V^{\prime}\right)^{-1}\left(y_{n}\right)=y_{n+1}-2 y_{n}+y_{n-1}, \quad n \in \mathbb{Z} .
$$

The main results of Ref. 12 can be formulated as follows. For $\omega_{b} \approx 2$ (maximal phonon frequency), all small amplitude solutions $y_{n}$ (even in $t$ ) have the form

$$
y_{n}(t)=b_{n} \cos \left(\omega_{b} t\right)+\varphi\left(t, b_{n-1}, b_{n}, \omega_{b}\right),
$$

where $\varphi$ is a smooth time-periodic function with frequency $\omega_{b} \quad$ and $\operatorname{Sup}_{t \in\left[0,2 \pi / \omega_{b}\right]}\left|\varphi\left(t, b_{n-1}, b_{n}, \omega_{b}\right)\right|=O\left(\left(\left|b_{n-1}\right|+\left|b_{n}\right|\right)\right.$ $\left.\times\left(\left|b_{n-1}\right|+\left|b_{n}\right|+\left|\omega_{b}-2\right|^{2}\right)\right)$. This result can be seen as an exact version of the rotating-wave approximation method, ${ }^{6}$ in the small amplitude limit. Note that $\varphi$ can be computed at an arbitrary order (see Ref. 13, p. 51). Moreover, $b_{n}$ satisfies the second order nonlinear recurrence relation

$$
b_{n+1}+2 b_{n}+b_{n-1}=-\mu b_{n}+B b_{n}^{3}+\text { h.o.t }
$$

where $\mu=\omega_{b}^{2}-4 \ll 1$ and the coefficient $B$ is given in (2). Higher order terms are $O\left(\left|b_{n}\right|\left(\left(\left|b_{n-1}\right|+\left|b_{n}\right|\right)^{2}+|\mu|\right)^{2}\right.$ ) [note that the right-hand side of (8) can be computed at an arbitrary order]. Consequently, the problem of finding small amplitude time-periodic solutions of (6) reduces to the problem (8), which can be viewed as a two-dimensional map $\left(b_{n}, b_{n-1}\right) \rightarrow\left(b_{n+1}, b_{n}\right)$.

For $B>0$ fixed and $\mu>0$ sufficiently small, the recurrence relation (8) has homoclinic solutions to 0 satisfying $\lim _{n \rightarrow \pm \infty} b_{n}=0$ [a proof has been given in Refs. 12 and 13, using the invariance $n \rightarrow-n$ of (8)]. More precisely, there exist homoclinic solutions to 0 denoted as $\pm b_{n}^{1}, \pm b_{n}^{2}$, having different symmetries $b_{-n-1}^{1}=-b_{n}^{1}, b_{-n}^{2}=b_{n}^{2}$.

Beyond this particular result, one can expect the existence of infinitely many homoclinic solutions to 0 (not necessarily symmetric). Indeed, for $B>0$ the intersections of the stable and unstable manifolds of $b_{n}=0$ are generically transverse, which yields the existence of "homoclinic tangles."22

A formal way to understand why homoclinic orbits exist is the following. Setting $\xi_{n}=(-1)^{n} b_{n}$ yields the recurrence relation

$$
\xi_{n+1}+\xi_{n-1}-2 \xi_{n}=\mu \xi_{n}-B \xi_{n}^{3}+\text { h.o.t. }
$$

Since $0<\mu \ll 1$, Eq. (9) can be approximated by an integrable differential equation

$$
v^{\prime \prime}=v-v^{3}
$$

with

$$
\xi_{n}=\sqrt{\frac{\mu}{B}} v(n \sqrt{\mu}) .
$$

Equation (10) has the homoclinic solutions $v(x)$ $= \pm \sqrt{2} / \cosh (x+c)$.

Using the above analysis, it has been proved that for $B>0$ and $\omega_{b}-2>0$ sufficiently small, there exist SAB solutions of (6) given by Eq. (7) and homoclinic solutions of (8) (see Refs. 12 and 13). SAB have the form

$$
y_{n}=(-1)^{n} \xi_{n} \cos \left(\omega_{b} t\right)+\text { h.o.t., }
$$

where $\xi_{n}=(-1)^{n} b_{n}$ satisfies the recurrence relation (9) and $\left|\xi_{n}\right| \rightarrow 0$ as $n \rightarrow \pm \infty$. The above homoclinic solutions $b_{n}^{1}, b_{n}^{2}$ correspond via Eq. (7) to $\mathrm{SAB} y_{n}^{1}, y_{n}^{2}$ having different symmetries $y_{-n-1}^{1}(t)=y_{n}^{1}\left(t+T_{b} / 2\right), y_{-n}^{2}=y_{n}^{2}$, where $T_{b}=2 \pi / \omega_{b}$ is the breather period (see Ref. 13, pp. 58 and 59). Note that $y_{n}\left(t+T_{b} / 2\right) \neq-y_{n}(t)$ in general due to higher order terms in (12) (however the egality holds if $V$ is even). Homoclinic solutions $-b_{n}^{i}$ of (8) simply correspond to $y_{n}^{i}\left(t+T_{b} / 2\right)$.

The exact solutions $y_{n}^{1}, y_{n}^{2}$ can be approximated at leading order using (10) and (11) with $v(x)=\sqrt{2} / \cosh x$. This yields

$$
y_{n}^{2}(t) \simeq(-1)^{n} \sqrt{\frac{2 \mu}{B}} \frac{\cos \omega_{b} t}{\cosh (n \sqrt{\mu})},
$$

and by symmetry we construct the second approximation 


$$
y_{n}^{1}(t) \simeq(-1)^{n} \sqrt{\frac{2 \mu}{B}} \frac{\cos \omega_{b} t}{\cosh ((|n+1 / 2|-1 / 2) \sqrt{\mu})} .
$$

Under these approximations one has $y_{n}^{i}\left(t+T_{b} / 2\right) \approx-y_{n}^{i}(t)$ and $y_{0}^{1}(t) \approx y_{0}^{2}(t)$. In fact, the exact solutions satisfy $y_{n}^{i}\left(t+T_{b} / 2\right)$ $=-y_{n}^{i}(t)+O(|\mu|)$ and $y_{0}^{1}(t)=y_{0}^{2}(t)+O\left(|\mu|^{3 / 2}\right)$ as $\mu \rightarrow 0^{+}$.

Notice that approximation (13) can also be derived using multiscale expansions. ${ }^{16}$ Moreover, these expressions also approximate $u_{n}$ since $u_{n}=y_{n}+O\left(y_{n}^{2}\right)$.

These calculations show that the maximum amplitude $A$ $\approx \sqrt{2 \mu / B}$ of the SAB is $O\left(\left|\omega_{b}-2\right|^{1 / 2}\right)$ as $w_{b} \rightarrow 2^{+}$. More precisely, breathers with amplitude $A \approx 0$ have frequency $\omega_{b}$ $\approx 2+(B / 8) A^{2}$.

Although SAB decay exponentially as $|n| \rightarrow+\infty$, their width is $O\left(\left|\omega_{b}-2\right|^{-1 / 2}\right)$ and diverges as $w_{b} \rightarrow 2^{+}$. As a consequence, if $B>0$ breathers exist for any small value of energy in the FPU system (1).

\section{B. Numerical continuation}

We have computed these solutions numerically in order to check the range of validity of approximations (13) and (14). We have fixed $K_{4}=1$ in Eq. (3) and thus the parameter $B$ is positive if $\left|K_{3}\right|<K_{3}^{*}=\sqrt{3} / 2 \simeq 0.86$.

We have performed our computations using a numerical scheme based on the concept of anticontinuous limit and path continuation with the Newton method. ${ }^{23}$ With this technique it is more convenient to use the difference displacements variables $u_{n}$. Indeed, with these new variables the dynamical equations become

$$
\ddot{u}_{n}+2 V^{\prime}\left(u_{n}\right)-C\left[V^{\prime}\left(u_{n+1}\right)+V^{\prime}\left(u_{n-1}\right)\right]=0, \quad n \in \mathbb{Z},
$$

where $C=1$ for our system, but it makes possible to consider the system (15) as a Klein-Gordon system, with an on-site potential $V$ and an anticontinuous limit at $C=0$. Note that $u_{n}$ is one-to-one related to the forces $y_{n}$ at small amplitudes, because $V^{\prime}$ is locally invertible since $V^{\prime \prime}(0) \neq 0$.

We use periodic boundary conditions $u_{n+2 p}(t)=u_{n}(t)$. The periodicity is considered for an even number of sites in order that the maximum frequency of the linear phonons is exactly 2 as in the infinite lattice (this frequency corresponds to the phonon with wave number $\pi$ ).

We have obtained breathers with symmetries $u_{n}(t)$ $=u_{-n}(t) \quad$ (site-centered mode) and $u_{n}(t)=u_{-n-1}\left(t+T_{b} / 2\right)$ (bond-centered mode). Note that the coordinates transformation (5) produces an exchange of the symmetry properties between both modes, i.e., in the difference displacements variables the site-centered mode is the Page mode ${ }^{7}$ and the bond-centered mode is the Sievers-Takeno mode. ${ }^{6}$

Figure 1 shows the excellent agreement between the approximation (13) (dashed line) and an exact site-centered mode obtained numerically (circles) for $\omega_{b}=2.01 \quad(\mu$ $\approx 0.04$ ).

We have continued the SAB obtained numerically for $B>0$ as $\omega_{b}$ goes away from the phonon band. In Fig. 2 we have plotted the maximum amplitude of the forces $\sup \left|y_{n}\right|$ (dashed lines) versus $\mu^{1 / 2}$ for two different values of the

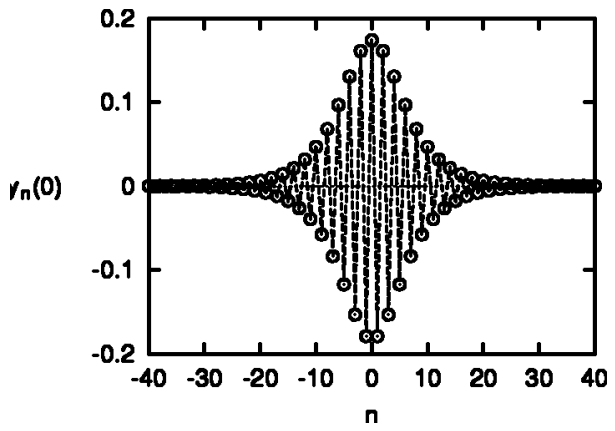

FIG. 1. Comparison between an exact site-centered mode (circles) and the approximation (13) (dashed line) for $\omega_{b}=2.01(\mu$ $\approx 0.04)$. The cubic coefficient of the potential is $K_{3}=-0.3(B$ $=2.64$ ), but similar results are obtained for any other fixed value of $K_{3}$ with $\left|K_{3}\right|<K_{3}^{*}(B>0)$ and $\mu>0$ small enough.

cubic coefficient, $K_{3}=-0.6(B=1.56)$ and $K_{3}=-0.3(B$ $=2.64$ ) from top to bottom, respectively. The maximum amplitude of the forces is an approximately linear function of $\mu^{1 / 2}$ up to $\mu \approx 0.6$. The continuous lines represent the maxima given by approximation (13). This approximation is not satisfactory for very small values of $B$ since higher order terms in (8) cannot be neglected when $B \rightarrow 0$. More precisely, the smaller $B$ is, the smaller $\mu$ must be chosen in (13) in order to have a good approximation.

The maximum amplitude of the relative displacements $\sup \left|u_{n}\right|$ is also an approximately linear function of $\mu^{1 / 2}$ (see squares in Fig. 2). This was expected for small $\mu$ since $u_{n}$ $=y_{n}+O\left(y_{n}^{2}\right)$, but it occurs far from the phonon band, at least until values of $\mu \approx 2.25$. In fact, we have checked that, surprisingly, expressions (13) and (14) fit very well the profile of the relative displacements $u_{n}$ even far from the phonon band as Fig. 3 shows. Note that the vibration amplitudes of the breather in Fig. 3 are quite large.

In Fig. 4 we have plotted again the maximum amplitude of the relative displacements (squares), but now for an even potential $\left(K_{3}=0\right)$ and larger frequencies, in order to compare approximation (14) with an approximation derived by Sievers and Takeno in the large amplitude regime. ${ }^{6}$ The SieversTakeno approximation is obtained using the rotating wave approximation (only the first Fourier component of solutions is taken into account) and Green's function techniques. For

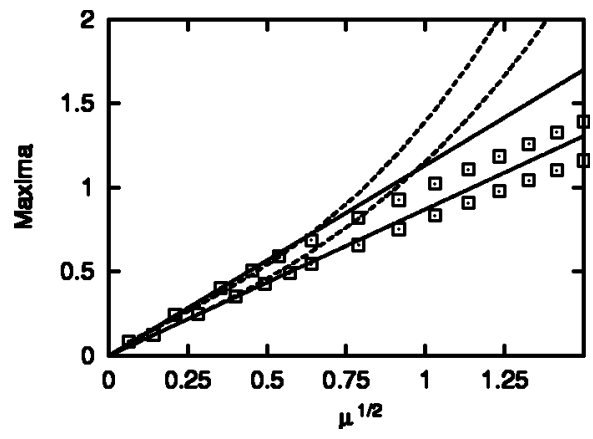

FIG. 2. Force (dashed line) and relative displacements (squares) maxima versus $\mu^{1 / 2}$. The continuous line corresponds to the maxima given by approximation (13). Parameters from top to bottom: $K_{3}=-0.6(B=1.56)$ and $K_{3}=-0.3(B=2.64)$. 


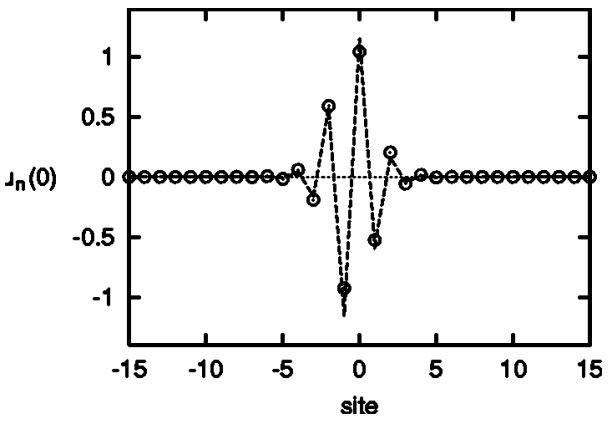

FIG. 3. Comparison between an exact bond-centered mode (circles) and the analytical approximation (14) (dashed line) for $\mu$ $=1.76$. The value of $\mu$ corresponds to a breather frequency $\omega_{b}$ $=2.4$ relatively far from the phonon band. The cubic coefficient of the potential $V$ is $K_{3}=-0.3(B=2.64)$.

the Sievers-Takeno mode and $K_{4}=1(B=3)$ it predicts

$$
\sup \left|u_{n}\right| \approx \frac{2}{3} \sqrt{w_{b}^{2}-3} .
$$

This amplitude-frequency relation corresponds to the dashed line in Fig. 4. On the other hand, from Eq. (14) it follows (continuous line):

$$
\sup \left|u_{n}\right| \approx \sqrt{\frac{2\left(w_{b}^{2}-4\right)}{3}} .
$$

Clearly we see that approximation (16) fits better very localized solutions with large amplitudes, whereas approximation (17) works better up to moderate amplitudes $\left(\omega_{b} \approx 2.4\right.$ in this case). In this sense both approximations can be considered complementary.

In addition we have performed a Floquet analysis in order to study the linear stability of the $\mathrm{SAB}^{24}$ In the symmetric potential case the result is well known: ${ }^{9}$ the Page mode is stable while the Sievers-Takeno mode has a harmonic instability (a pair of real eigenvalues $\sigma, \sigma^{-1}$ close to 1) that increases with the breather frequency. When a cubic term in the potential $V$ is introduced $\left(K_{3} \neq 0\right)$, the situation is more complex. The Sievers-Takeno mode shows again a harmonic instability [see Fig. 5(a)], but it also shows oscillatory instabilities. As Fig. 5(b) shows for the Page mode, these oscilla-

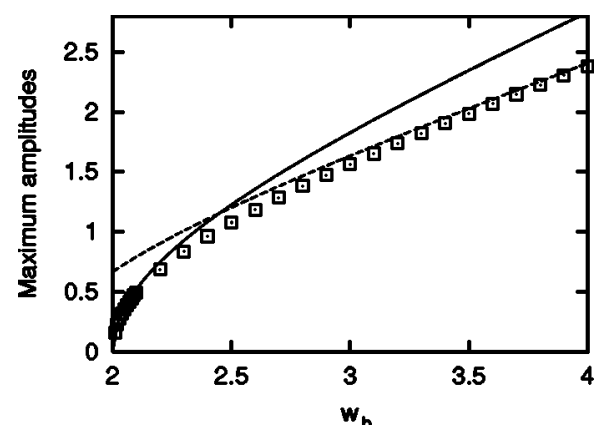

FIG. 4. Maximum amplitudes, $\sup \left|u_{n}\right|$, of the Sievers-Takeno mode versus frequency for an even potential. The continuous line is the maxima given by Eq. (17), while the dashed line corresponds to the Sievers-Takeno approximation. Squares correspond to the numerically computed solution.
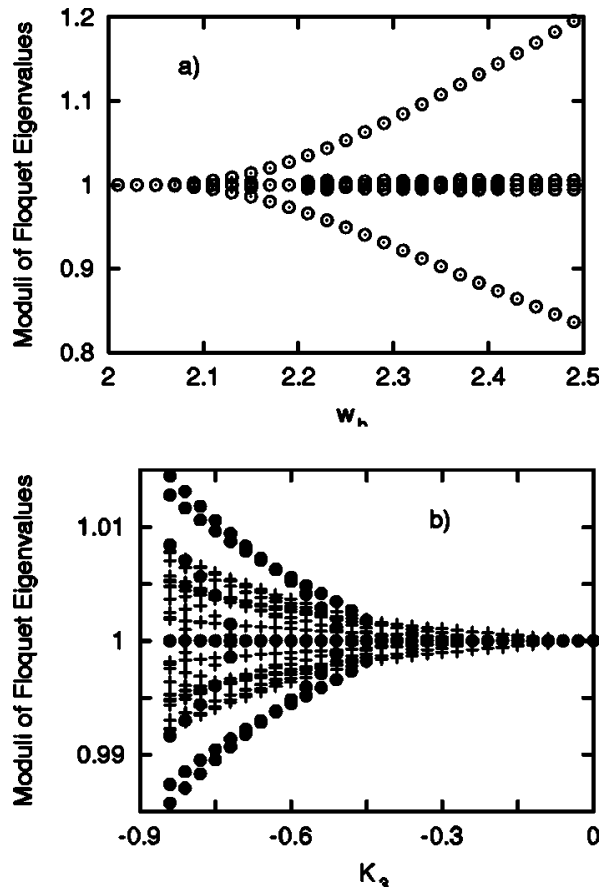

FIG. 5. (a) Harmonic instability of the Sievers-Takeno mode for $K_{3}=-0.3$; (b) Oscillatory instabilities of the Page mode versus $K_{3}$ for a lattice with 52 sites (full circles) and a lattice with 100 sites (pluses). The frequency is $w_{b}=2.1$.

tory instabilities increase with $\left|K_{3}\right|$. Although most of them are size dependent effects due to the discretization of continuous spectrum, ${ }^{25}$ for the infinite system some of them might remain out of the unit circle and in this case SAB would be unstable. We shall not examine this problem here and leave it for future works.

\section{LARGE AMPLITUDE BREATHERS WITH AN ENERGY THRESHOLD}

In this section we study breathers in the parameter region $B<0$. Using the recurrence relation (8), one can show that $\mathrm{SAB}$ with frequencies slightly above the phonon band do not exist for $B<0$ (see Refs. 12 and 13). However, large amplitude breathers do exist in this parameter region. ${ }^{11}$ More precisely, for $V$ strictly convex $\left(K_{4}>0,\left|K_{3}\right|<\sqrt{3 K_{4}}\right)$ and $B<0$ $\left(\sqrt{3 K_{4}} / 2<\left|K_{3}\right|\right)$, breathers whose amplitudes do not tend to zero as $w_{b} \rightarrow 2^{+}$have been numerically obtained. ${ }^{11,21,27}$ As a consequence there is an energy threshold for breathers creation in these FPU systems. The criterion $\sqrt{3 K_{4}} / 2<\left|K_{3}\right|<\sqrt{3 K_{4}}$ for the existence of an energy threshold originates from Ref. 21 , and follows by combining analytical results in Ref. 13 (nonexistence of small amplitude breathers) and Ref. 11 (existence of large amplitude breathers).

The existence of an energy threshold for breather creation has been formally analyzed by Flach, Kladko, and MacKay ${ }^{18}$ for general Hamiltonian systems, in a different case when small amplitude breathers bifurcate at an edge of the phonon spectrum. An energy threshold exists above a critical lattice dimension $d$ (typically $d=2$ ) which depends on the nonlinear 


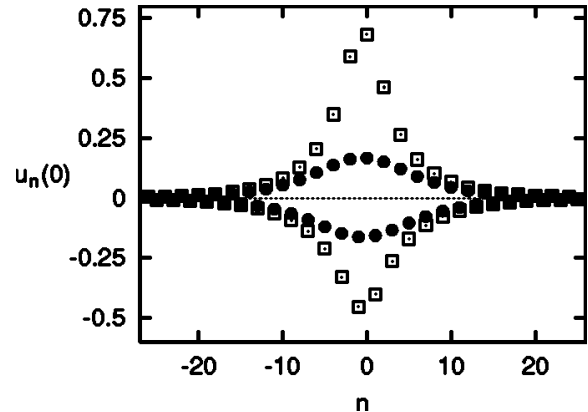

FIG. 6. Comparison between a small amplitude breather (full circles) for $B>0$ and a large amplitude breather (squares) for $B<0$ with the same frequency $w_{b}=2.01$. Parameters: SAB $K_{3}=-0.3$, $K_{4}=1(B=2.64)$; LAB $K_{3}=-1, K_{4}=1(B=-1)$.

terms of the Hamiltonian. This analysis has been extended to a class of nonanalytic Hamiltonian systems in Ref. 27. The existence of excitation thresholds has been rigorously analyzed by Weinstein ${ }^{19}$ for the $d$-dimensional discrete nonlinear Schrödinger equation, using variational methods (see also Ref. 20 for formal variational arguments in the onedimensional case).

In Fig. 6 we compare a SAB and a LAB with the same frequency $w_{b}=2.01$. As it is shown in Fig. 7(a) the energy of a LAB (dashed line) does not go to zero when approaching the phonon band edge. It lies always above a certain positive lower bound. In the case of SAB (continuous lines) the breather energy can be lowered to arbitrarily small values.
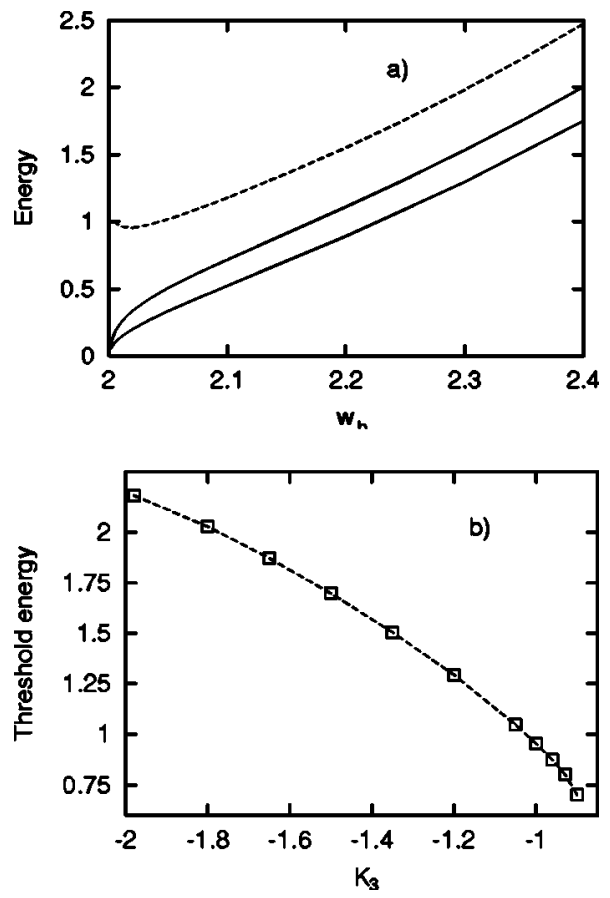

FIG. 7. (a) Breather energy versus frequency close to the phonon band edge (we fix $K_{4}=1$ ). The dashed line shows the existence of a threshold in energy for a LAB with $K_{3}=-1(B<0)$. The continuous lines ( $K_{3}=-0.6$ and $K_{3}=0$ from top to bottom) show that for $B>0 \mathrm{SAB}$ of arbitrary low energy exist. (b) Dependence of the LAB energy threshold on the asymmetry parameter $K_{3}$.

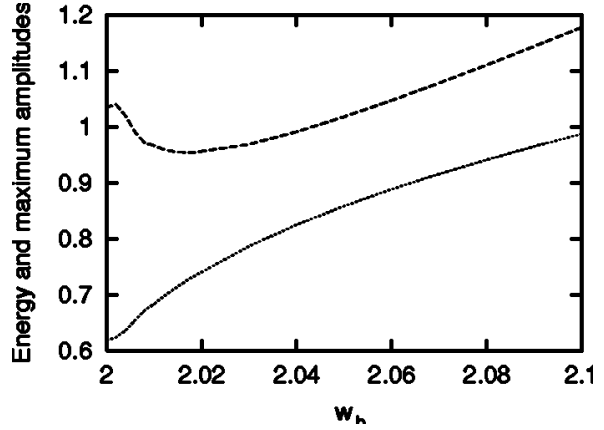

FIG. 8. LAB energy (upper line) and amplitude (lower line) vs frequency close to the phonon band edge (we fix $K_{3}=-1, K_{4}=1$ ).

The dependence of the energy threshold on the asymmetry parameter $K_{3}$ is also shown in Fig. 7(b), where we have fixed $K_{4}=1$. One observes that the energy threshold goes to 0 as $B \rightarrow 0^{-}$(for $K_{3} \rightarrow-\sqrt{3} / 2 \approx-0,86$ ). Moreover, LAB families also exist for nonconvex potentials $\left(K_{3}<-\sqrt{3} \approx-1,73\right)$, and their energy threshold increases with $\left|K_{3}\right|$. This shows that the criterion $\sqrt{3 K_{4}} / 2<\left|K_{3}\right|<\sqrt{3 K_{4}}$ for the existence of an energy threshold is not optimal. We conjecturate that an energy threshold appears under the more general condition $K_{4}>0, \sqrt{3 K_{4}} / 2<\left|K_{3}\right|(B<0)$ in the one-dimensional FPU system.

Figure 8 shows the energy of a LAB (upper line) and its amplitude (lower line) versus frequency, close to the phonon band edge [the upper line is a close-up of Fig. 7(a) near $\omega_{b}$ $=2]$. The reason for the nonmonotonous behavior of energy is that there are two competing tendencies: on the one hand amplitudes are decreasing with frequency and going to a nonzero lower bound, but on the other hand the width of the breather is increasing and more and more particles begin to oscillate as $\omega_{b}$ decreases.

We have found LAB with the same symmetries as SAB (Page and Sievers-Takeno modes). LAB have an exponential decay for $\omega_{b}>2$. As Fig. 9 shows, we have been able to fit the LAB profiles to the following expression in the case of the Page mode:

$$
u_{n}(t) \approx \alpha\left(\omega_{b}\right) \sigma\left(\omega_{b}\right)^{|n|} \cos \left(\omega_{b} t\right),
$$

where

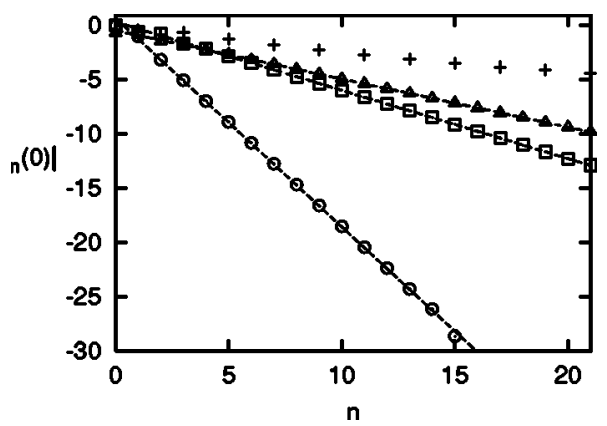

FIG. 9. Logarithms of the Page mode profiles for $K_{3}=-1, K_{4}$ $=1$ and $w_{b}=3$ (circles), $w_{b}=2.1$ (squares), $w_{b}=2.05$ (triangles) and $w_{b}=2$ (pluses). The dashed line is the logarithm of Eq. (18) after fitting the parameter $\alpha$. Note that Eq. (18) does not work in the limit $\omega_{b}=2$. 


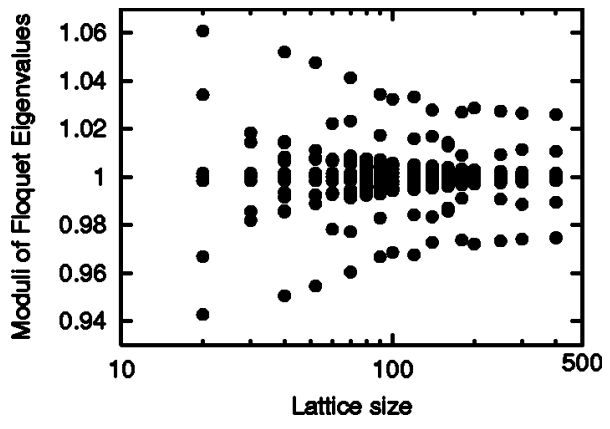

FIG. 10. Floquet eigenvalues of the Page mode for $\omega_{b}=3, K_{3}$ $=-1$ and $K_{4}=1$, depending on system size. The eigenvalue of largest modulus and its inverse seem to converge to values out of the unit circle for large system sizes.

$$
\sigma\left(\omega_{b}\right)=1-\frac{\omega_{b}^{2}}{2}+\frac{\omega_{b}}{2}\left(\omega_{b}^{2}-4\right)^{1 / 2} \in(-1,0),
$$

and $\alpha\left(\omega_{b}\right)$ is a fitting parameter. A similar expression corresponds to the Sievers-Takeno mode.

We have computed the decay rate $\left|\sigma\left(\omega_{b}\right)\right|$ as follows. The FPU system (1) can be formulated as a mapping

$$
\left(\begin{array}{c}
y_{n} \\
y_{n+1}
\end{array}\right)=F\left(\begin{array}{c}
y_{n-1} \\
y_{n}
\end{array}\right)
$$

where the map

$$
F\left(\begin{array}{c}
y_{n-1} \\
y_{n}
\end{array}\right)=\left(\begin{array}{c}
y_{n} \\
\frac{d^{2}}{d t^{2}}\left(V^{\prime}\right)^{-1}\left(y_{n}\right)+2 y_{n}-y_{n-1}
\end{array}\right)
$$

acts in a space of smooth time-periodic functions with frequency $\omega_{b}>2$. The linearized operator

$$
D F(0)\left(\begin{array}{c}
y_{n-1} \\
y_{n}
\end{array}\right)=\left(\begin{array}{c}
y_{n} \\
\left(\frac{d^{2}}{d t^{2}}+2\right) y_{n}-y_{n-1}
\end{array}\right)
$$

has a purely hyperbolic spectrum and $\sigma\left(\omega_{b}\right), \sigma\left(\omega_{b}\right)^{-1}$ are the closest eigenvalues to -1 [with $\sigma(2)=-1$ ].

We have observed that LAB remain localized for $\omega_{b}=2$ (see plus signs in Fig. 9) and thus approximation (18) does not work in this limit.

The stability properties of LAB are the same as SAB with non-even potentials, i.e., both modes show oscillatory instabilities and also a harmonic instability in the case of the Sievers-Takeno mode. As we mention in Sec. II, most of the oscillatory instabilities are size dependent effects due to the discretization of continuous spectrum. ${ }^{25}$ However, a band analysis ${ }^{11}$ suggests that, at least for some parameter values (close to $\omega_{b}=3, K_{3}=-1, K_{4}=1$ ), some eigenvalues remain out of the unit circle for the infinite system and LAB are unstable. We have studied the evolution of Floquet eigenvalues of the Page mode for $\omega_{b}=3, K_{3}=-1$ and $K_{4}=1$ when the system size becomes larger. In Fig. 10 one can see that one pair of eigenvalues seems to converge to values out of the unit circle for large system sizes, as stated in Ref. 11.

\section{DARK BREATHERS}

Using the recurrence relation (8), it has been shown that for $B<0$ and $\mu<0$ small enough ( $\omega_{b}$ lies in the phonon band) there exist small amplitude heteroclinic solutions of (6) connecting two nonlinear normal modes $y_{n}^{ \pm}$at infinity, i.e., $\lim _{n \rightarrow \pm \infty} y_{n}(t)-y_{n}^{ \pm}(t)=0$ (see Refs. 12 and 13 ).

We now briefly describe the nonlinear normal modes $y_{n}^{ \pm}$. These solutions are spatially periodic $\left[y_{n+2}^{ \pm}(t)=y_{n}^{ \pm}(t)\right]$, have 0 time-average (the mean interaction force is 0 ), and neighbors oscillate out of phase $\left[y_{n+1}^{ \pm}(t)=y_{n}^{ \pm}\left(t+T_{b} / 2\right)\right]$. Moreover, solutions $y_{n}^{ \pm}$are equal up to a half-period phase shift $\left[y_{n}^{+}(t)\right.$ $\left.=y_{n}^{-}\left(t+T_{b} / 2\right)\right]$. Note that the corresponding displacement patterns $x_{n}$ are in general different from the zone-boundary modes $x_{n}(t)=(-1)^{n} f(t)$ (also called binary oscillations). Indeed, in this latter case the time average of relative displacements $u_{n}$ is 0 , whereas interaction forces have 0 time average in our case. However, both types of solutions coincide for even potentials $V$.

To obtain an approximate expression of the heteroclinic solutions, we can rewrite the recurrence relation (9) in the following form:

$$
\frac{\rho_{n+1}+\rho_{n-1}-2 \rho_{n}}{-\mu}=-\rho_{n}-B \rho_{n}^{3}+\text { h.o.t. }
$$

using the variable change $\xi_{n}=\sqrt{-\mu} \rho_{n}$. Equation (23) can be approximated by the differential equation,

$$
v^{\prime \prime}=-v-B v^{3}
$$

with $\rho_{n}=v(n \sqrt{\mu})$. Consequently, the system (6) has a family of small amplitude solutions which can be approximated by

$$
y_{n}(t) \simeq(-1)^{n} \sqrt{\frac{\mu}{B}} \tanh \left(\frac{n \sqrt{-\mu}}{\sqrt{2}}\right) \cos \left(\omega_{b} t\right) .
$$

This type of solutions are called dark breathers ${ }^{28}$ or antisymmetric kinks. ${ }^{29}$ Typically the term breathers refers to bright breathers, that is a few excited units in a lattice. Here we have the opposite kind of localization: most oscillators are excited except one or a few of them.

Expression (25) approximates exact site-centered solutions of (6) satisfying $y_{-n}(t)=y_{n}\left(t+T_{b} / 2\right)$. Under our approximation one has $y_{n}\left(t+T_{b} / 2\right) \approx-y_{n}(t)$, and in fact the exact solutions satisfy $y_{n}\left(t+T_{b} / 2\right)=-y_{n}(t)+O(|\mu|$ ) (uniformly in $n \in \mathbb{Z}$ ) as $\mu \rightarrow 0^{+}$.

Equation (6) has also exact bond-centered dark breather solutions satisfying $y_{n}(t)=y_{-n+1}(t)$. We shall approximate them by

$$
y_{n}(t) \simeq(-1)^{n} \sqrt{\frac{\mu}{B}} \tanh \left(\frac{(-n+1 / 2) \sqrt{-\mu}}{\sqrt{2}}\right) \cos \left(\omega_{b} t\right) .
$$

Note that expressions (25) and (26) also approximate $u_{n}$ since $u_{n}=y_{n}+O\left(y_{n}^{2}\right)$.

For computing dark breathers numerically we consider a lattice with an odd number of sites (51 lattice sites). We first choose an even potential $\left(K_{3}=0\right)$ with $K_{4}<0$ (so that $B<0$ ). We solve Eq. (15) in Fourier space by the Newton method, 


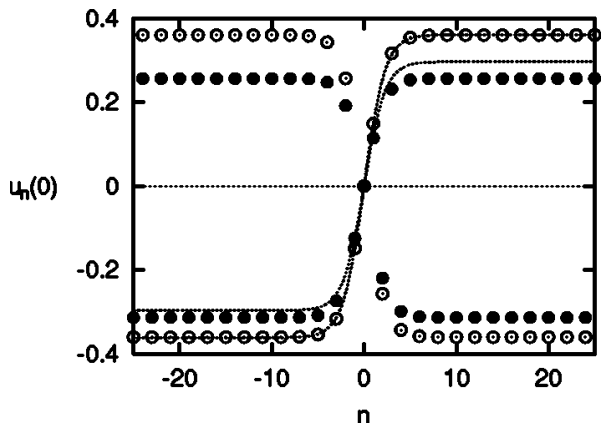

FIG. 11. Small amplitude site-centered dark breathers for a frequency $\omega_{b}=1.9$. The circles represent an exact solution computed numerically with an even potential $\left(K_{4}=-1\right)$, while the full circles were obtained adding to the previous potential a cubic term with a coefficient $K_{3}=0.6$. These solutions have the symmetry $u_{-n}(t)$ $=u_{n}\left(t+T_{b} / 2\right)$. The dashed lines are the tanh function of approximation (25) for both cases.

fixing the time average of $u_{0}$ to 0 and using (25) as an initial condition. Dark breathers are computed for non-even potentials by continuation with respect to $K_{3}$, starting from $K_{3}$ $=0$ (during the continuation the time average of $y_{n}$ remains very small, due to the constraint on $u_{0}$ ).

Figure 11 shows the excellent agreement between an exact site-centered solution obtained numerically (circles) and the approximation (25) (dashed line), for an even potential with $K_{4}=-1(B=-3)$ and a frequency $\omega_{b}=1.9$. For non-even potentials (full circles) the agreement is not so good. The reason is clearly that the approximation of $u_{n}$ by $y_{n}$ with Eq. (25) has the symmetry property $u_{n}\left(t+T_{b} / 2\right)=-u_{n}(t)$ which is not true any more when $K_{3} \neq 0$.

Similar results are obtained for bond-centered solutions (see Fig. 12).

For a fixed potential we can continue numerically these solutions decreasing $\omega_{b}$. As Fig. 13 shows, then the oscillation amplitudes increase and for even potentials expression (25) continues matching very well the relative displacements $u_{n}$ (circles), even far from the top of the phonon band. For non-even potentials (squares) the agreement is also excellent close enough to the edge of the phonon band. For lower frequencies we observe discrepancies becoming larger.

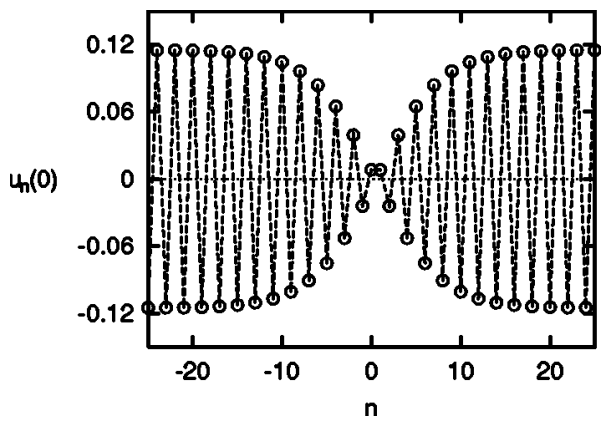

FIG. 12. Small amplitude bond-centered dark breather for an even potential with $K_{4}=-1$ and a frequency $\omega_{b}=1.99$. The circles represent an exact solution computed numerically, having the symmetry $u_{n}(t)=u_{-n+1}(t)$. The dashed line corresponds to the analytical approximation (26).

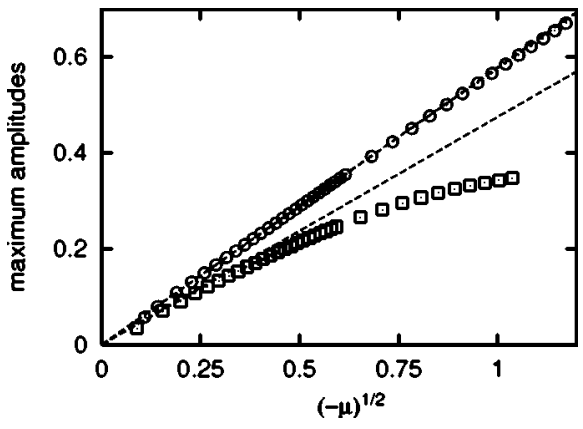

FIG. 13. Maximum amplitudes, sup $\left|u_{n}\right|$, of the site-centered dark breather versus $-\mu^{1 / 2}$ for $K_{3}=0$ (circles) and $K_{3}=0.6$ (squares). In both cases $K_{4}=-1$. The dashed line is the maxima given by Eq. (25). In the non-even case approximation (25) does not provide a good fitting far from the phonon band edge.

We have also performed a Floquet analysis to investigate the linear stability of this family of solutions when we decrease the frequency.

On the one hand, the site-centered mode is stable close to the edge of the phonon band for even potentials as Fig. 14(a) shows. However, a cascade of harmonic instabilities appears for low enough frequencies (larger amplitudes). These instabilities are visible in Fig. 14(a) at $\omega_{b} \approx 1.43$. Before the appearance of the harmonic instabilities one observes an instability bubble at $\omega_{b} \approx 1.53$. Nevertheless, its magnitude decreases as the system size increases and thus it should not be relevant for the infinite system.

On the other hand, the bond-centered mode shows a harmonic instability [see Fig. 15(a)] that increases when the frequency decreases. One also observes another cascade of harmonic instabilities at $\omega_{b} \approx 1.43$.
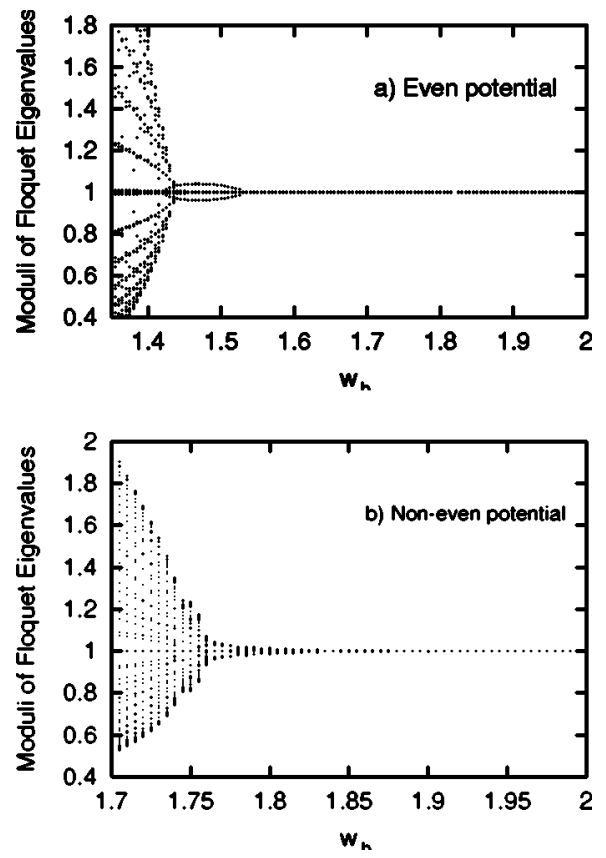

FIG. 14. Evolution of the Floquet eigenvalue moduli versus frequency for the site-centered dark breather. (a) Even potential $\left(K_{3}\right.$ $=0$ ); (b) non-even potential $\left(K_{3}=-0.6\right)$. In both cases $K_{4}=-1$. 

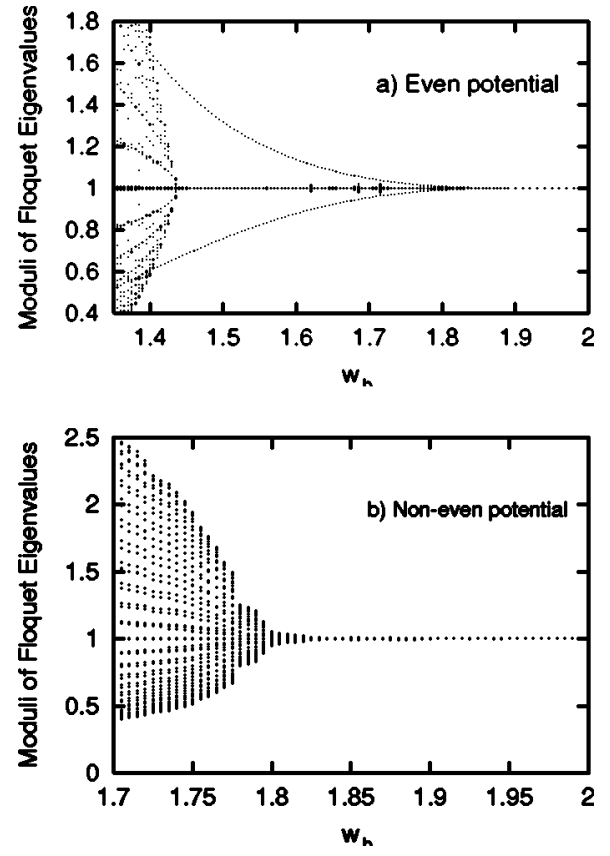

FIG. 15. Evolution of the Floquet eigenvalue moduli versus frequency for the bond-centred dark breather. (a) Even potential $\left(K_{3}\right.$ $=0)$; (b) Non-even potential $\left(K_{3}=-0.6\right)$. In both cases $K_{4}=-1$.

Non-even potentials induce oscillatory instabilities for low enough frequencies (larger amplitudes), both for the sitecentered and the bond-centered modes. Figure 14(b) and 15(b) show that this occurs for frequencies lower than 1.85, for $K_{3}=-0.6$ and $K_{4}=-1$. Numerically we have not found these oscillatory instabilities near the phonon band edge, but we do not exclude that they might exist and be harder to detect.

The harmonic instability before mentioned for the bondcentered mode is also present for non-even potentials, but is not visible in Fig. 15(b) due to oscillatory instabilities. Note that frequency scales in Figs. 15(a) and 15(b) are different. For this reason in Fig. 15(b) we do not see either the cascade of harmonic instabilities around $\omega_{b} \approx 1.43$. We have checked that they are also present with non-even potentials.

\section{BRIGHT AND DARK BREATHERS WITH UNIFORM STRESS}

Breathers considered in Sec. III for $B<0$ can be numerically continued at fixed frequency up to values $B>0$, bringing about a new type of solutions which satisfy $\lim _{n \rightarrow \pm \infty} u_{n}$ $=c$, where $c$ is a nonzero constant. As Fig. 16 shows, these new type of solutions can coexist with $\mathrm{SAB}$ for the same values of the parameters and can be seen as time-periodic oscillations around static solutions of (1) given by $x_{n}=c n$. The lattice is uniformly stretched at infinity for $c>0$, and uniformly compressed for $c<0$. Such breather solutions with a constant static strain $c<0$ have been also observed by Sandusky and Page in the case $B<0$ (see Ref. 26, p. 876).

This section provides analytical and numerical results concerning breather solutions with a constant static strain. The case of dark breathers with uniform stress is also examined.

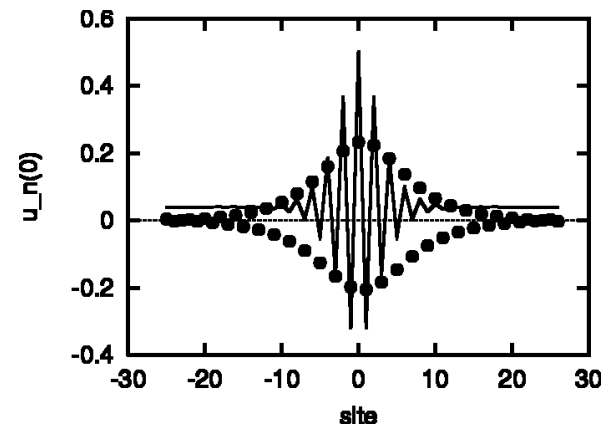

FIG. 16. Comparison between a SAB (full circles) and a breather with a uniform stress (continuous line) for the same values of the parameters: $K_{3}=-0.6, K_{4}=1, w_{b}=2.01$.

In the sequel we assume $V^{\prime \prime}(c)>0$. We make the change of variable $x_{n}(t)=c n+\tilde{x}_{n}\left(t \sqrt{V^{\prime \prime}(c)}\right)$ where $\tilde{x}_{n} \ll 1$ and introduce the modified potential

$$
\tilde{V}(u)=\left(V(c+u)-V^{\prime}(c) u\right) / V^{\prime \prime}(c) .
$$

Equation (1) leads to

$$
\frac{d^{2}}{d t^{2}} \tilde{x}_{n}=\tilde{V}^{\prime}\left(\tilde{x}_{n+1}-\tilde{x}_{n}\right)-\tilde{V}^{\prime}\left(\tilde{x}_{n}-\tilde{x}_{n-1}\right), \quad n \in \mathbb{Z},
$$

with $\tilde{V}^{\prime}(0)=0$ and $\tilde{V}^{\prime \prime}(0)=1$. One can see that Eq. (28) is exactly Eq. (1) where $V$ has been changed to $\widetilde{V}$. Consequently, the results of the above sections (both analytical and numerical) readily apply to Eq. (28).

Returning to the original variables, the following analytical results for the FPU system (1) follow from the analysis of Sec. II A. Let us fix $c \in \mathbb{R}$ and define

$$
B(c)=\frac{1}{2} V^{\prime \prime}(c) V^{(4)}(c)-\left(V^{(3)}(c)\right)^{2} .
$$

If $B(c)>0$ and $\omega_{b}-2 \sqrt{V^{\prime \prime}(c)}>0$ is sufficiently small, the FPU system has a family of exact solutions which can be approximated by

$$
u_{n}(t) \simeq c+(-1)^{n} \sqrt{\frac{2 \mu}{B(c)}} \frac{\cos \left(\omega_{b} t\right)}{\cosh \left(n \sqrt{\mu / V^{\prime \prime}(c)}\right)},
$$

where $\mu=\omega_{b}^{2}-4 V^{\prime \prime}(c), 0<\mu \ll 1$. These solutions are time periodic (with frequency $\omega_{b}$ ) and their oscillating part is spatially localized. As $n \rightarrow \pm \infty$ they converge towards a uniformly stressed static state (stretched or compressed depending on $c$ ). Note that their lower frequency lies inside the phonon band if $V^{\prime \prime}(c)<1$, and above for $V^{\prime \prime}(c)>1$. The exact solutions approximated by (30) are site-centered, i.e., they satisfy $u_{n}(t)=u_{-n}(t)$. There exists also exact bond-centered solutions satisfying $u_{n}(t)=u_{-n-1}\left(t+\pi / \omega_{b}\right)$. In the sequel we shall denote these solutions as breathers with uniform stress.

As we previously mentioned, the numerical study of these solutions is already contained in Sec. II B, which applies to the renormalized displacements $\tilde{x}_{n}$ satisfying (28). For the polynomial potential (3), breathers with uniform stress have the same stability properties as the SAB corresponding to the modified potential (27). In particular, oscillatory instabilities are observed for $K_{3}=0$ ( $V$ is even), since the modified poten- 
tial $\widetilde{V}$ is non-even in this case. On the contrary, if $K_{3} \neq 0$, $K_{4}>K_{3}^{2} / 6$ then the modified potential $\tilde{V}$ is even for $c=-K_{3} / 3 K_{4}$ [and the conditions $V^{\prime \prime}(c)>0, B(c)>0$ are realized]. In this case oscillatory instabilities are not observed for the SAB with specific static strain $c$.

If $B(c)<0$ and $K_{4}>0$, we numerically obtain large amplitude solutions of the same type, whose oscillating parts do not vanish as $w_{b} \rightarrow 2 \sqrt{V^{\prime \prime}(c)}$ [with $w_{b}>2 \sqrt{V^{\prime \prime}(c)}$ ]. This result follows directly from the numerical study of Sec. III, as well as the stability properties of these solutions. These LAB with uniform stress have been previously observed by Sandusky and Page (see Ref. 26, pp. 874-876), for the interaction potential (3) with $B<0$. In their examples $V^{\prime \prime}(c)>1$ (since $c<0, K_{3}<0, K_{4}>0$ ) and thus the lower frequencies of these LAB families lie strictly above the phonon band. This phenomenon can be observed in Fig. 8 of Ref. 26, which provides frequency vs amplitude plots of such solutions (note that $c$ may not be constant on each curve).

If $B(c)<0$ and $\omega_{b}-2 \sqrt{V^{\prime \prime}(c)}<0$ is sufficiently small, the FPU system has a family of exact solutions which can be approximated by

$$
u_{n}(t) \simeq c+(-1)^{n} \sqrt{\frac{\mu}{B(c)}} \tanh \left(\frac{n \sqrt{-\mu}}{\sqrt{2 V^{\prime \prime}(c)}}\right) \cos \left(\omega_{b} t\right),
$$

where $\mu=\omega_{b}^{2}-4 V^{\prime \prime}(c)<0,|\mu| \ll 1$. These solutions are time periodic (with frequency $\omega_{b}$ ) and consists in a dark breather superposed with a uniformly stressed static state. Note that their higher frequency lies above the phonon band if $V^{\prime \prime}(c)>1$. These solutions are site-centred $\left[u_{-n}(t)=u_{n}(t\right.$ $\left.+\pi / \omega_{b}\right)$ ], but their bond-centered analogues also exist. They have the same stability properties as dark breathers in Sec. IV, for the modified potential (27).

\section{CONCLUSIONS}

In this work we have analyzed both numerically and analytically a rich variety of nonlinear solutions in FPU lattices. There are four main results.
On the one hand, we have numerically explored the range of validity of a recent breathers (bright or dark) existence proof $^{12}$ for one-dimensional FPU lattices. Our computations have been carried out with polynomial interaction potentials of degree 4. For $B>0$, we have found that the maximum amplitude of bright breathers in the relative displacement variables is an approximately linear function of $\mu^{1 / 2}$ even far from the phonon band and, in fact, the approximate expression (14) describes very well the profile of the breathers when their amplitudes are large. For $B<0$ and $K_{4}<0$, we find in the same way that dark breathers can be very well described by approximate analytical expressions.

On the other hand, we have found numerically for $B<0$ large amplitude bright breathers having frequencies arbitrary close to the top of the phonon band, which are out of range of the local analysis. ${ }^{12}$ This breather family exhibits an energy threshold, which is a rarely observed phenomenon in one-dimensional lattices.

Moreover, with respect to stability properties we have checked that non-even potentials induce oscillatory instabilities in both bright and dark cases. In the case of even potentials, the Page mode is linearly stable while the SieversTakeno mode is unstable. We have also found that bondcentered dark breathers are unstable. The site-center dark breather is unstable for low enough frequencies and linearly stable with an even potential near the edge of the phonon band.

At last, we have analyzed small amplitude bright and dark breathers superposed to uniformly stressed static states $x_{n}$ $=c n$. In particular, the lowest frequency of bright breathers is inside the phonon band if $0<V^{\prime \prime}(c)<1$, and the highest frequency of dark breathers is above the phonon band if $V^{\prime \prime}(c)>1$. We have obtained local conditions on $V$ for their existence as well as approximate analytical expressions.

\section{ACKNOWLEDGMENTS}

This work has been supported by the European Union under the RTN project LOCNET, HPRN-CT-1999-00163. J. Cuevas acknowledges a FPDI grant from la Junta de Andalucía. G. James acknowledges stimulating discussions with R. MacKay.
${ }^{1}$ S. Flach and C. R. Willis, Phys. Rep. 295, 181 (1998).

${ }^{2}$ A. J. Sievers and J. B. Page, in Dynamical Properties of Solids, edited by G. K. Horton and A. A. Maradudin (North-Holland, Amsterdam, 1995), Vol. 7 Chap. 3, p. 137.

${ }^{3}$ R. S. Mackay and S. Aubry, Nonlinearity 7, 1623 (1994).

${ }^{4}$ R. Livi, M. Spicci, and R. S. Mackay, Nonlinearity 10, 1421 (1997).

${ }^{5}$ S. Flach, Phys. Rev. E 51, 1503 (1995).

${ }^{6}$ A. J. Sievers and S. Takeno, Phys. Rev. Lett. 61, 970 (1988).

${ }^{7}$ J. B. Page, Phys. Rev. B 41, 7835 (1990).

${ }^{8}$ G. Huang, Z. Shi, and Z. Xu, Phys. Rev. B 47, 14561 (1993).

${ }^{9}$ S. R. Bickham, S. A. Kiselev, and A. J. Sievers, Phys. Rev. B 47, 14206 (1993).
${ }^{10}$ K. W. Sandusky, J. B. Page, and K. E. Schmidt, Phys. Rev. B 46, 6161 (1992).

${ }^{11}$ S. Aubry, G. Kopidakis, and V. Kadelburg, Discrete Contin. Dyn. Syst. 1, 271 (2001).

${ }^{12}$ G. James, C. R. Acad. Sci., Ser. I: Math. 332, 581 (2001).

${ }^{13}$ G. James, J. Nonlinear Sci. 13, 27 (2003).

${ }^{14} \mathrm{G}$. James and P. Noble, in Proceedings of the Third Conference on Localization and Energy Transfer in Nonlinear Systems, San Lorenzo de El Escorial, Spain, 17-21 June 2002, edited by L. Vázquez, R. S. MacKay, and M. P. Zorzano (World Scientific, Singapore, 2003).

${ }^{15}$ P. Noble, Nonlinearity $\mathbf{1 7}, 803$ (2004).

${ }^{16}$ A. Tsurui, Prog. Theor. Phys. 48, 1196 (1972). 
${ }^{17}$ S. Flach, Physica D 91, 223 (1996).

${ }^{18}$ S. Flach, K. Kladko, and R. S. MacKay, Phys. Rev. Lett. 78, 1207 (1997).

${ }^{19}$ M. I. Weinstein, Nonlinearity 12, 673 (1999).

${ }^{20}$ B. Malomed and M. I. Weinstein, Phys. Lett. A 220, 91 (1996).

${ }^{21}$ B. Sánchez-Rey, J. F. R. Archilla, G. James, and J. Cuevas, in Proceedings of the Third Conference on Localization and Energy Transfer in Nonlinear Systems, San Lorenzo de El Escorial, Spain, 17-21 June 2002, edited by L. Vázquez, R. S. MacKay, and M. P. Zorzano (World Scientific, Singapore, 2003).

${ }^{22}$ J. Guckenheimer and P. Holmes, Nonlinear Oscillations, Dynamical Systems, and Bifurcations of Vector Fields (Springer-Verlag, New York, 1983).

23 J. L. Marin and S. Aubry, Nonlinearity 9, 1501 (1996); J. L.
Marín, Ph.D. dissertation, University of Zaragoza, Department of Condensed Matter, 1997.

${ }^{24}$ S. Aubry, Physica D 103, 201 (1997).

${ }^{25}$ J. L. Marin and S. Aubry, Physica D 119, 163 (1998).

${ }^{26}$ K. W. Sandusky and J. B. Page, Phys. Rev. B 50, 866 (1994).

${ }^{27}$ M. Kastner, Phys. Rev. Lett. 92, 104301 (2004).

28 A. Alvarez, J. F. R. Archilla, J. Cuevas, and F. R. Romero, New J. Phys. 4, 72 (2002); A. M. Morgante, M. Johansson, G. Kopidakis, and S. Aubry, Physica D 162, 53 (2002); Yu. S. Kivshar, W. Krolikowski, and O. A. Chubykalo, Phys. Rev. E 50, 5020 (1994).

${ }^{29}$ V. Bortolani, A. Franchini, and R. F. Wallis, Phys. Rev. B 56, 8047 (1997). 